\title{
PERCEPCIONES DEL RIESGO ASOCIADO A LA PRÁCTICA DEL MOTOTAXISMO EN CARTAGENA, COLOMBIA
}

\author{
FRANCISCO JAVIER MAZA ÁVILA, MARÍA PAULA FALS GALEZO, \\ LAURA CRISTINA ESPINOSA FLÓREZ, CAMILA FERNANDA SAFAR \\ CANO Y DANIELA LICONA DÁGER \\ Universidad de Cartagena, Cartagena, Colombia
}

\section{RESU MEN}

El objetivo fundamental de este artículo consiste en analizar los factores de riesgo asociados al oficio de mototaxista en Cartagena. Otro objetivo es el de estudiar la influencia que estos factores tienen en la percepción de los mototaxistas respecto a sufrir lesiones causadas por accidentes de tránsito. Los datos relacionados con los factores de riesgo y las percepciones sobre lesiones causadas por el tránsito, se obtuvieron de una encuesta estructurada construida por el equipo de investigadores, a partir de referencias de la Organización Internacional del Trabajo, la Organización Mundial de la Salud y de la comunidad académica, y aplicada a un total de 403 personas que ejercen el oficio de mototaxista en Cartagena, asumiendo una población infinita y tomando como referencia un nivel de confianza del $95 \%$ y un error muestral del 4,9\%. Los resultados arrojan que el oficio del mototaxismo lleva a quienes lo ejercen a estar constantemente expuestos a altos niveles de riesgos ambientales, físicos y psicológicos. Otro resultado fue que un gran porcentaje de los mototaxistas reconocen que el oficio representa un gran riesgo que los expone a accidentes de tránsito; sin embargo, se ven en la obligación de ejercer esta actividad al carecer de suficientes oportunidades de conseguir otro tipo de empleos.

\footnotetext{
Este artículo es uno de los productos obtenidos del proyecto de investigación titulado "Factores de riesgo asociados a la accidentalidad entre mototaxistas de Cartagena de Indias", financiado por la Universidad de Cartagena en la Quinta convocatoria para la financiación de proyectos de semilleros de investigación (No 056-2017). Correo electrónico: Francisco Maza Ávila, fmazaa@unicartagena.edu.co. Recibido: 25 de septiembre de 2019; aceptado: 27 de noviembre de 2019.
}

Econáma\& Regón Vd. 13, Nb 2, (Gartagena, dicientre 2019), pp 57-81 
Palabras claves: Transporte informal, mototaxismo, factores de riesgo, accidentalidad, condiciones de salud, percepción.

Clasificaciones JEL: C12, C16, I18, J01, R41

\section{ABST RACT}

\section{Perception of safety issues related to the operation of motorcycle taxi services in Cartagena, Colombia}

We aim to study the safety issues related to motorcycle informal taxi services in Cartagena. We also aim to understand the motorcycle-operators view of these issues in relation to the risk of getting injured. Data related to safety issues and traffic injuries was obtained from a unique dataset gathered by implementing a structured survey intended to follow the designs suggested by the International Labor Organization, the World Health Organization and the academic literature. We surveyed 403 motorcycle taxi operators in Cartagena, assuming an infinite population, a 95\% confidence interval and a sample error of $4,9 \%$. The results show that providing motorcycle taxi services leaves its operators permanently exposed to high levels of environmental, physical, and psychological safety issues. Another result was that a large percentage of them are aware that operating motorcycles represents a large risk, in addition to leaving them exposed to sustaining traffic accidents; nonetheless, they feel compelled to stay at the occupation, as there are insufficient job alternatives available to them.

Keywords: Informal transport, informal job, mototaxista, risk factors, accidents, health conditions, perception.

JEL Classification: C12, C16, I18, J01, R41

\section{INTRODUCCIÓN}

El transporte informal de pasajeros en motocicletas en Colombia -conocido comúnmente en la región Caribe como "mototaxi"- nació a principios de la década de los noventas como respuesta al alto desempleo de las zonas marginales 
del departamento de Córdoba (Brieva et al., 2011). En la actualidad, el servicio de mototaxi es el medio de transporte ilegal más usado por diversas poblaciones del país -es común en, al menos, 26 de los 32 departamentos de Colombia- y se ha convertido en el medio de supervivencia laboral para personas que, en su mayoría, son de escasos recursos (Castillo et al., 2013).

Cartagena es una de las ciudades del Caribe colombiano con el mayor número de personas ejerciendo el oficio de mototaxismo (Maza et al., 2019). Según las cifras del Observatorio Nacional de Seguridad Vial, al 31 de marzo de 2019 estaban registradas en Cartagena 68.660 motocicletas; sin embargo, el número que circulan en la ciudad se estima que es considerablemente mayor, teniendo en cuenta aquellas que provienen de otros municipios tanto del departamento de Bolívar como de la región Caribe. De hecho, se estima que su número supera las 80.000 motocicletas. Asimismo, la cantidad de personas cuyos ingresos directos dependen del mototaxismo en Cartagena es de alrededor de 90.000 (Maza et al., 2019).

El mototaxismo cuenta con una alta aceptación entre los habitantes de Cartagena. Por una parte, en 2018 los resultados de la Encuesta de Percepción Ciudadana (Cartagena Cómo Vamos 2019), mostraban que el 28\% de los desplazamientos diarios de los cartageneros se hacían en motocicletas -sean propias o empleadas para servir de mototaxis- mientras que un $28 \%$ lo realizaba en buses o busetas y otro 20\% empleaba el Sistema Integrado de Transporte Masivo, Transcaribe. Por otra parte, el 70,4\% de los cartageneros se sentía satisfecho con el servicio de moto o mototaxis (promedio ponderado entre ambas alternativas), frente a un nivel de satisfacción del 53\% con el servicio de Transcaribe y del $42 \%$ con el servicio de buses o busetas. Posibles razones que explican la anterior situación se relacionan con la rapidez, los bajos costos, el servicio "puerta a puerta" y la falta de opciones de transporte entre ciertos tramos de la ciudad (Brieva et al., 2011; Jiménez et al., 2013).

El mototaxismo parece minimizar el problema de movilidad para los cartageneros. Sin embargo, al mismo tiempo se constituye en un problema social: el incremento de motocicletas dedicadas a esta labor en las vías genera un mayor riesgo para quienes se ocupan en este oficio. Para 2018, según las cifras del Observatorio Nacional de Seguridad Vial, del total de lesionados y muertos en accidentes de tránsito en Cartagena, el 58,26\% y el 56,98\%, respectivamente, eran usuarios de motocicletas; vale señalar que las cifras superan los promedios nacionales para ese mismo año (56,03\% y 50,48\%, respectivamente). Un mayor número de accidentes, junto con enfermedades causadas por la exposición constante a los cambios climáticos e inadecuadas condiciones para el ejercicio 
de su labor conducen a un desequilibrio en el estado de salud de la persona y, por ende, a una mala calidad de vida (Castillo et al., 2013). La situación anterior advierte que el mototaxismo es un tema de contrastes: por un lado, es una alternativa de subsistencia y minimización del déficit de transporte público, pero por otra parte es fuente de problemas sociales.

Teniendo en cuenta lo anterior, este artículo de investigación tiene como objetivo fundamental examinar los factores de riesgo asociados al oficio de mototaxista en Cartagena, así como la incidencia que estos factores tienen en la percepción de lesiones causadas por el tránsito ${ }^{1}$ por parte de quienes lo ejercen, de manera que la información arrojada pueda servir de base para la formulación de recomendaciones dirigidas a prevenir o mitigar dichos riesgos. Se espera que los resultados de este trabajo despierten el interés de la comunidad en general y que contribuyan en la búsqueda de la mejor solución al problema del mototaxismo en la ciudad.

\section{REVISIÓN DEL ESTADO DEL ARTE}

El transporte informal es una actividad que suscita interés en todo el mundo. En África, Asia y Latinoamérica, la búsqueda de alternativas diferentes al transporte público autorizado es un fenómeno que ha sido estudiado desde diversos ámbitos; por ejemplo, dentro de los trabajos de Madougou et al. (2016) en Benin, África, los de Barboza et al. (2014) y Ruesga et al. (2014) en Brasil y los de Quistberg (2014) en Perú, el común denominador es el uso de motocicletas, prohibidas en estos países por los riesgos que conlleva su uso, principalmente para el conductor o "mototaxista". Entre los riesgos asociados se encuentran la exposición a condiciones perjudiciales para la salud: sustancias químicas como el benceno, y al monóxido de carbono, el riesgo de accidentalidad que se ve incrementado por el ejercicio, la falta de uso del equipamiento y la violación de normas de tránsito, siendo esta última muy influyente.

En Colombia, el estudio del transporte informal ha encontrado que el oficio del mototaxismo es un medio de transporte aceptado socialmente, pese a su ilegalidad y a los altos riesgos de accidentalidad para sus usuarios. El trabajo de Castillo (2010) en Montería arroja, por ejemplo, que esta actividad informal es

\footnotetext{
${ }^{1}$ Según la Organización Mundial de la Salud, una lesión o traumatismo causado por el tránsito es una lesión, sea o no letal, que se ha producido como resultado de una colisión en la vía pública en la que se ha visto implicado, al menos, un vehículo en movimiento. Los niños, peatones, ciclistas y personas de edad avanzada son los usuarios más vulnerables de las vías de tránsito.
} 
un estímulo para la economía local. En este mismo sentido están los trabajos de Espinosa et al. (2008b) para Cundinamarca, e Hinestroza-Filigrana et al. (2015) para el Valle del Cauca, aunque se evidencian las condiciones de vulnerabilidad para quienes lo ejercen, destacando que gran parte de los mototaxistas no se encuentran afiliados al régimen de seguridad social, restringiendo el acceso a beneficios fundamentales de un trabajo digno, y exponiéndose a riesgos físicos y ergonómicos.

El transporte público colombiano -en especial, el ofertado en la región Caribe- se caracteriza por su insuficiencia y poca calidad, lo que lleva al incremento de demanda de alternativas como el mototaxismo y, por lo tanto, el incremento de ofertantes, que usualmente son motociclistas con poca experiencia, lo que conlleva al exceso de velocidad y a arriesgadas maniobras de adelantamiento, a vías urbanas fluidas donde se fomenta altas velocidades, al aumento de la interacción entre vehículos, y a la falta de mantenimiento de la infraestructura. Aun cuando no se encontraron estudios puntuales de la efectividad de las medidas del Gobierno Nacional para el control del transporte informal, sí se han considerado propuestas de alternativas de servicio de transporte público formal de pasajeros en equipos no automotores como el bicitaxi, en Bogotá, que garantizaría seguridad y comodidad para los usuarios.

En Cartagena existe falta de control del uso de mototaxis y, en general, de las motocicletas. Los estudios de Castillo et al. (2013) y Maza et al. (2019) muestran que las motocicletas representan el flujo principal de tráfico en la ciudad; asimismo, las normativas no han sido suficientes para disminuir el ejercicio del mototaxismo, pues medidas como el "Pico y Placa" han tenido el efecto opuesto sobre el número de motocicletas para trabajar y la ganancia esperada, que en ambos casos aumenta considerablemente, ya que los mototaxistas optan por emplear una motocicleta adicional. En el Cuadro 1 se referencian algunas de las principales investigaciones que abordan el oficio de transporte informal tanto a nivel internacional como nacional, a partir de la búsqueda efectuada en bases de datos como ScienceDirect y EbscoHost y repositorios universitarios, además de la suministrada por organismos oficiales de Cartagena, encargados de la regulación de la movilidad. 


\section{CUADRO 1}

\section{Antecedentes sobre el transporte informal mediante motocicletas}

\begin{tabular}{|c|c|c|}
\hline Año & Autores & Objetivo \\
\hline 2019 & Maza et al. (2019) & $\begin{array}{l}\text { Examinar los factores asociados al oficio de } \\
\text { mototaxista en Cartagena y su influencia en la } \\
\text { percepción de lesiones causadas por el tránsito, } \\
\text { como base para la formulación de recomendaciones } \\
\text { dirigidas a su prevención. }\end{array}$ \\
\hline 2017 & Maza et al. (2017) & $\begin{array}{l}\text { Estudiar la dinámica del transporte informal presente } \\
\text { en los municipios de Arjona, Turbaco, El Carmen de } \\
\text { Bolívar y Magangué, Colombia, los mecanismos y } \\
\text { acciones de los entes departamentales y municipales } \\
\text { para su regulación y las apreciaciones que tienen sus } \\
\text { habitantes tanto de los efectos económicos, sociales } \\
\text { y ambientales que ha traído dicha dinámica a su } \\
\text { municipio, como de la efectividad de las acciones } \\
\text { para su control. }\end{array}$ \\
\hline 2017 & Hothem et al. (2016) & $\begin{array}{l}\text { Evaluar el impacto clínico de la derogación que tuvo } \\
\text { lugar en Michigan en 2012, sobre la ley universal del } \\
\text { casco. }\end{array}$ \\
\hline 2016 & $\begin{array}{l}\text { Quiñones-Bolaños et al. } \\
\text { (2016) }\end{array}$ & $\begin{array}{l}\text { Desarrollar un modelo equivalente de ruido de } \\
\text { tráfico para simular el nivel de presión sonora } \\
\text { promedio en las intersecciones de carreteras con base } \\
\text { en las características del flujo del tráfico y del sitio, en } \\
\text { Cartagena, Colombia. }\end{array}$ \\
\hline 2016 & Madougou et al. (2016) & $\begin{array}{l}\text { Evaluar la incidencia y las consecuencias de los } \\
\text { accidentes de tráfico entre y sobre los conductores de } \\
\text { mototaxi de Cotonou, ciudad mayor de Benín. }\end{array}$ \\
\hline 2014 & Barbosa et al. (2014) & $\begin{array}{l}\text { Determinar la prevalencia de lesiones faciales y del } \\
\text { absentismo en mototaxistas que son víctimas de } \\
\text { accidentes de tránsito en una ciudad del noreste de } \\
\text { Brasil. }\end{array}$ \\
\hline 2014 & Quistberg et al. (2014) & $\begin{array}{l}\text { Examinar la relación entre choques de peatones con } \\
\text { motocicletas y la presencia de señales de tránsito } \\
\text { visibles para ambas partes, así como la temporización } \\
\text { de la señal para determinar su efectividad en la } \\
\text { seguridad del peatón, en Lima, Perú. }\end{array}$ \\
\hline
\end{tabular}

Fuente: Elaboración del autor. 


\section{CUADRO 1 (Continuación)}

Antecedentes sobre el transporte informal mediante motocicletas

\begin{tabular}{|c|c|l|}
\hline Año & Autores & \multicolumn{1}{c|}{ Objetivo } \\
\hline 2014 & Yánez y Acevedo (2014) & $\begin{array}{l}\text { Mostrar un registro de la tasa de infracción de la } \\
\text { norma de pico y placa por parte de los mototaxistas } \\
\text { de Cartagena, Colombia. }\end{array}$ \\
\hline 2013 & Castillo et al. (2013) & $\begin{array}{l}\text { Determinar las condiciones de trabajo y salud de los } \\
\text { mototaxistas de Cartagena. }\end{array}$ \\
\hline 2011 & Herazo et al. (2011) & $\begin{array}{l}\text { Demostrar el nivel de dependencia funcional } \\
\text { como medida de la discapacidad en un grupo de } \\
\text { mototaxistas debido a los accidentes de tránsito a los } \\
\text { que éstos están expuestos en Cartagena. }\end{array}$ \\
\hline 2006 & Arias (2005) & $\begin{array}{l}\text { Estudiar el uso de la motocicleta como medio de } \\
\text { transporte en Santiago de Cuba. }\end{array}$ \\
\hline
\end{tabular}

Fuente: Elaboración del autor.

\section{METODOLOGÍA}

Este artículo, de naturaleza y alcance explicativo, tuvo como objetivo poder conocer los principales factores de riesgos asociados al oficio del mototaxismo, así como la percepción que sobre las lesiones causadas por el tránsito tienen quienes lo ejercen en la ciudad. Los datos relacionados con los factores de riesgo y percepciones sobre lesiones causadas por el tránsito se obtuvieron de una encuesta estructurada diseñada por el equipo de investigación, a partir de los referentes de la Organización Internacional del Trabajo, la Organización Mundial de la Salud y de la literatura académica disponible. El cuestionario fue aplicado, mediante la técnica de encuesta, a una muestra de 403 personas que ejercen el oficio de mototaxista en Cartagena, a finales de 2017, asumiendo una población infinita y tomando como referencia un nivel de confianza del 95\% y un error muestral del 4,9\%.

La muestra se distribuyó de forma estratificada, en 15 puntos de concentración de mototaxistas, tomando como criterio para su selección aquellos lugares reconocidos por concentrar -a manera de estaciones- transportadores informales para ejercer su oficio, tales como el Centro de Cartagena, sector 
India Catalina; Barrio el Espinal, sector Castillo San Felipe; Barrio Escallón Villa, frente al Centro Comercial Los ejecutivos; Barrio el Bosque, en la vía que conduce a la zona industrial de Mamonal; Barrio La Castellana, frente al Centro Comercial Paseo de la Castellana; y barrio El Pozón, vía Terminal de Trasporte, entre otros. También se incluyeron tres estaciones del Sistema Integrado de Transporte Masivo -SITM- Transcaribe (María Auxiliadora, Cuatro Vientos y Madre Bernarda) y el llamado Corredor Universitario, donde se ubican las sedes Piedra de Bolívar y Zaragocilla de la Universidad de Cartagena, así como las sedes España y Cedesarrollo de la Fundación Universitaria Tecnológico Comfenalco (Mapa 1).

El proceso de selección de participantes por cada una de las estaciones se realizó aleatoriamente, otorgando la oportunidad a cada uno de los elementos observacionales a participar en la muestra. ${ }^{2}$ Además, y atendiendo a la dinámica del oficio de mototaxismo en Cartagena de Indias, al momento de la distribución de los cuestionarios, se le concedió un mayor peso a los días de la semana (de lunes a viernes) frente al fin de semana, así como un mayor peso a la jornada del día frente a la de la noche (considerando una jornada de trabajo de 16 horas) (Cuadro 2).

\section{MAPA 1}

Distribución de la aplicación de cuestionarios a mototaxistas en Cartagena

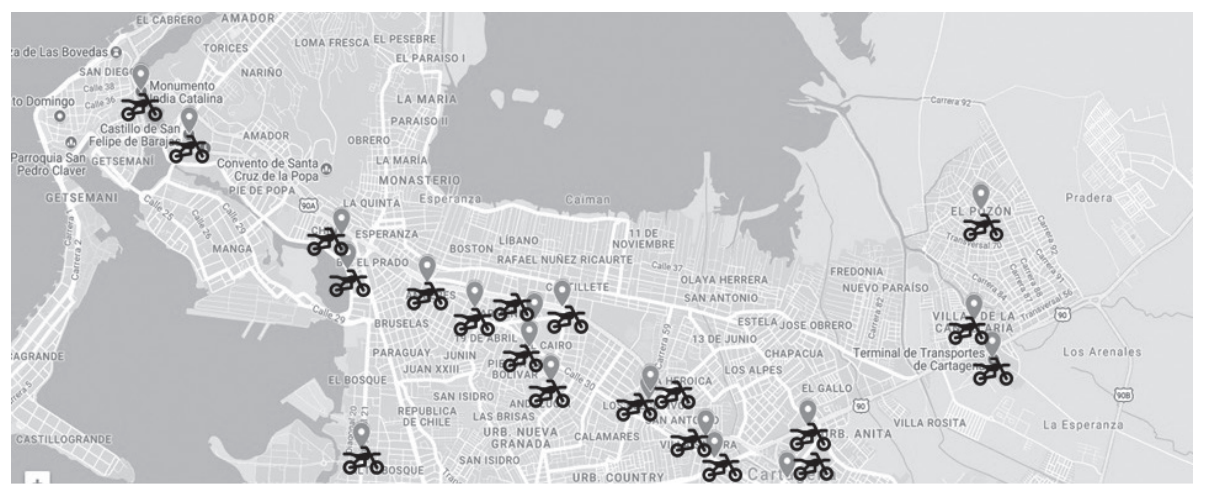

Fuente: Elaboración del autor.

\footnotetext{
${ }^{2}$ Vale señalar que cada punto de concentración no sólo es atendido por un personal fijo de mototaxistas (como sí podría ocurrir en una estación de taxis), sino que puede llegar cualquier persona que preste la actividad. De allí que haya sido posible esperar que se cumpla el supuesto de aleatoriedad, considerando tanto el día de la semana como la hora de aplicación del cuestionario.
} 


\section{CUADRO 2}

Consideración para la aplicación de cuestionarios a mototaxistas en Cartagena

\begin{tabular}{|c|c|c|}
\hline Distribución en la semana & Días & $\%$ \\
\hline Días de semana & 5 & $71,4 \%$ \\
\hline Fin de semana & 2 & $28,6 \%$ \\
\hline Total & 7 & $100 \%$ \\
\hline Distribución en un día de semana & Horas & $\%$ \\
\hline Día & 12 & $75,0 \%$ \\
\hline Noche & 4 & $25,0 \%$ \\
\hline Total & 16 & $100 \%$ \\
\hline
\end{tabular}

Fuente: Elaboración del autor.

\section{RESULTADOS}

\section{A. Caracterización socioeconómica del oficio de mototaxismo en Cartagena}

El examen de los resultados obtenidos a partir del cuestionario aplicado a mototaxistas de Cartagena, indica que la gran mayoría de personas que se dedican a esta actividad informal son hombres (99,01\%), aunque se observa una presencia minoritaria de mujeres. En este oficio predominan los adultos jóvenes, que son aquellos que se encuentran en el rango de edad entre 18 y 34 años (56,58\%) (Gráfico 1).

Varios aspectos de la caracterización se destacan a continuación. Se halló que el porcentaje más alto con respecto al nivel socioeconómico es el correspondiente al Estrato 1, que pertenece a la categoría más baja en la escala (50,13\%). También se encontró que un poco menos de la mitad de los mototaxistas viven en unión libre con sus parejas sentimentales (44,92\%), el 23,82\% se encuentra casado y el $28,2 \%$, soltero (Gráfico 1). Cabe señalar que la mayoría de los mototaxistas son oriundos del departamento de Bolívar, específicamente de Cartagena $(64,27 \%)$. Este lugar resulta ser también el municipio donde reside casi la totalidad de mototaxistas $(90,57 \%)$. 


\section{GRÁFICO 1}

Características sociodemográficas. Mototaxistas de Cartagena

Panel a: rango de edad

\begin{tabular}{cc}
\hline \multicolumn{1}{c}{ Edad } & $\%$ \\
\hline Menor de edad & $0.99 \%$ \\
Adulto joven & $56.58 \%$ \\
Adulto medio & $42.18 \%$ \\
Adulto mayor & $0.25 \%$ \\
\hline
\end{tabular}

Panel c: estado civil

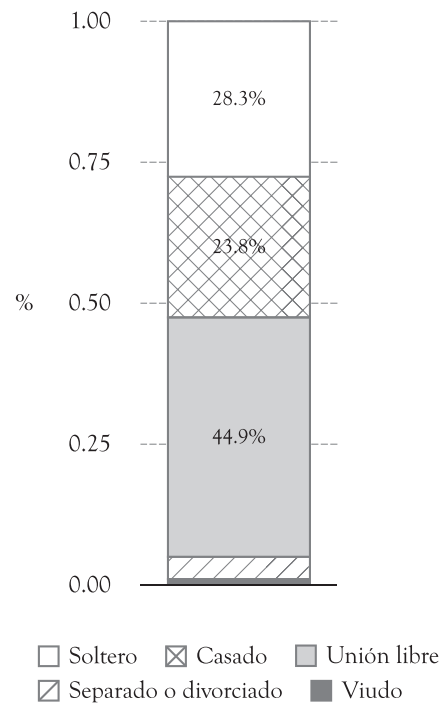

Panel b: género

\begin{tabular}{cr}
\hline Genero & \multicolumn{1}{c}{$\%$} \\
\hline Femenino & $0.99 \%$ \\
Masculino & $99.01 \%$ \\
\hline
\end{tabular}

Panel d: estrato socioeconómico

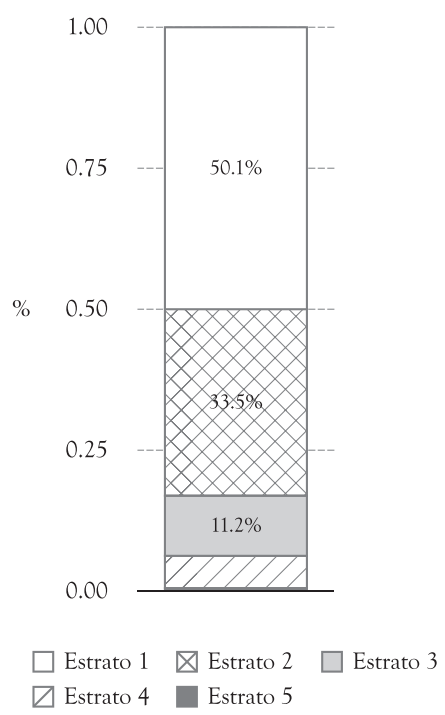

Fuente: Elaboración propia, a partir de cuestionario aplicado a mototaxistas.

Las personas que prestan este servicio, por lo general presentan un bajo nivel de formación académica. En efecto, el nivel más alto de escolaridad alcanzado para el 59,06\% de ellos es la secundaria, sin embargo, tan sólo el 20,10\% logró graduarse de bachiller. De hecho, un pequeño porcentaje han afirmado 
haber cursado algún tipo de estudios superiores, el 11,17\% ha culminado un programa técnico profesional y un $5,46 \%$ un programa tecnológico (Gráfico 2). Este resultado concuerda con lo expresado por Espinosa, Rodriguez y Uribe (2008, p.11), quienes afirman que “(...) el mototaxismo tambien surge como una oportunidad para las personas desempleadas, reinsertadas, analfabetas y personas con bajos niveles de educacion, bajo la modalidad de 'ecomomia del rebusque".

\section{GRÁFICO 2}

Nivel de escolaridad. Mototaxistas de Cartagena

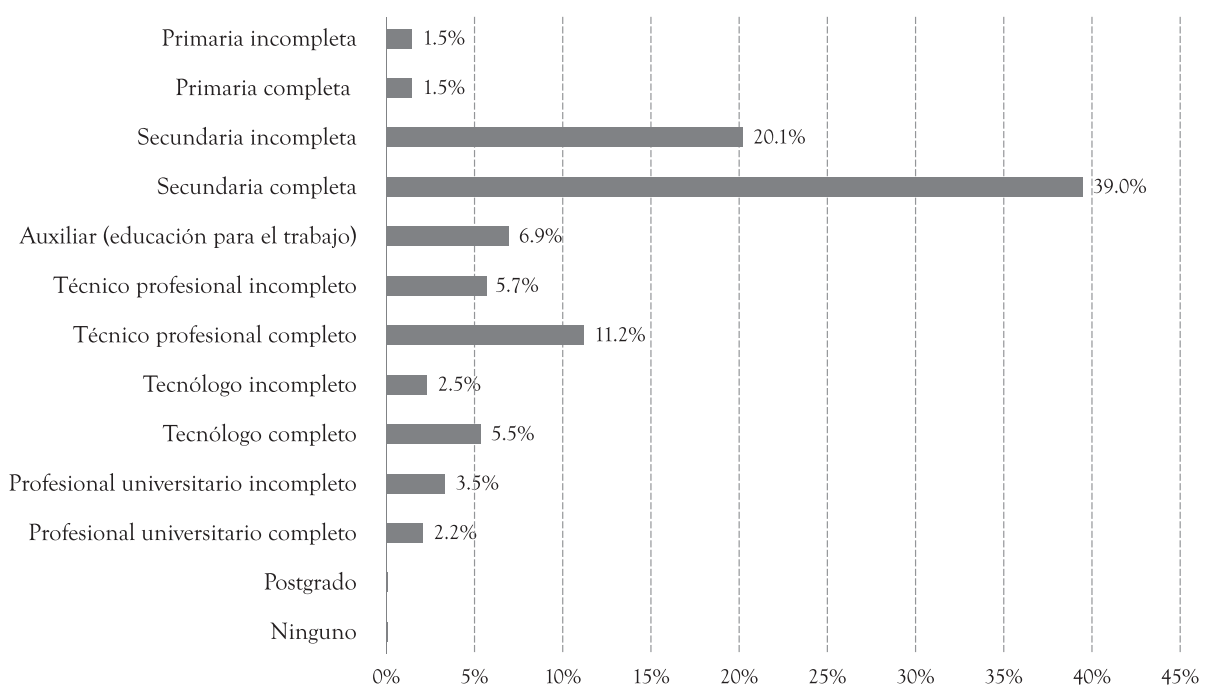

Fuente: Elaboración propia, a partir de cuestionario aplicado a mototaxistas

La mayoría de los mototaxistas llevan pocos años ejerciendo el oficio. De hecho, casi la mitad de ellos $(40,10 \%)$ sólo lleva entre 0 y 4 años. No obstante, se puede destacar también que el 6,51\% de ellos llevan más de 10 años (Gráfico 3). Vale la pena subrayar que existe asociación entre los años de dedicación y los rangos de edad de quienes ejercen el mototaxismo. ${ }^{3}$

\footnotetext{
${ }^{3}$ Prueba $\chi 2$, con un nivel de significancia del 5\% y con 18 grados de libertad, P-valor dedicación =0,00.
} 


\section{GRÁFICO 3}

Años de dedicación y edad. Mototaxistas de Cartagena

Panel a: años de dedicación

al oficio

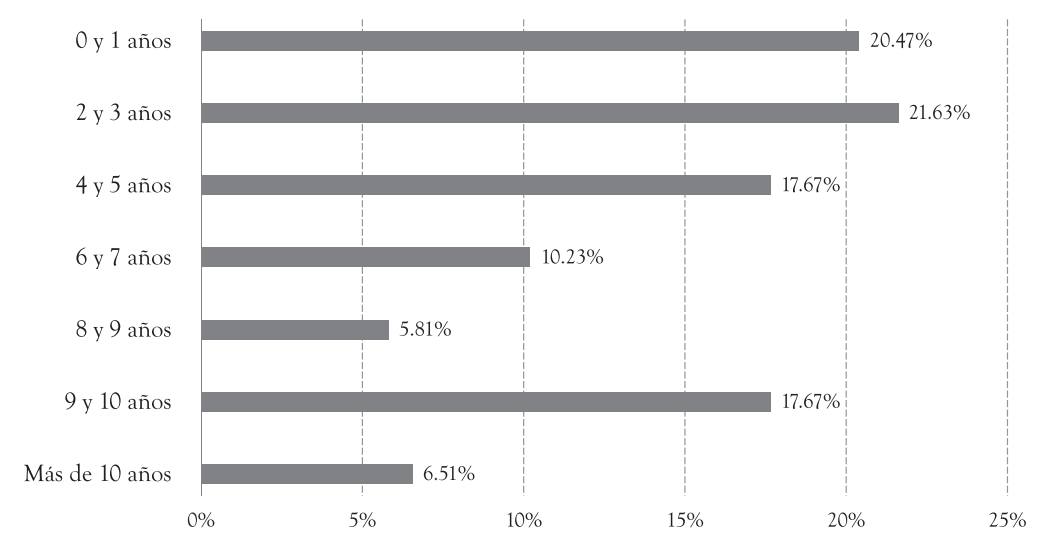

Panel b: años de dedicación

según edad

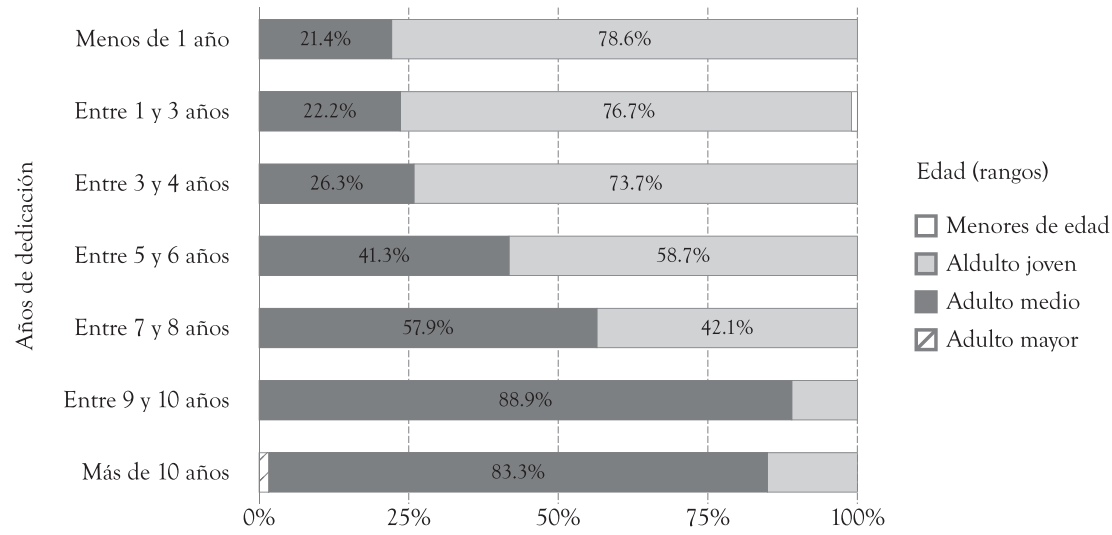

Fuente: Elaboración propia, a partir de cuestionario aplicado a mototaxistas.

Los mototaxistas de Cartagena en su mayoría le dedican toda la semana (75,68\%), incluyendo los fines de semana o los días festivos $(85,61 \%)$. De esta 
manera, para la mayoría de ellos, esta es la única actividad remunerada que desempeñan (73,80\%), además de ser su principal fuente de ingresos (90,92\%) (Gráfico 5). Una de las explicaciones más relevantes para este resultado es lo afirmado por Sánchez (2011, p. 13):

“(...) Esta actividad representa una respuesta eficaz para mitigar y reducir el desempleo que caracteriza las regiones donde el fenómeno se ha tornado particularmente fuerte, haciendo énfasis en la falta de oportunidades laborales como el principal factor responsable en generar la reproducción e incrementar la probabilidad de permanecer en el mismo"

\section{GRÁFICO 4 \\ Dedicación al oficio. Mototaxistas de Cartagena}

Panel a: frecuencia del ejercicio del mototaxismo en la semana

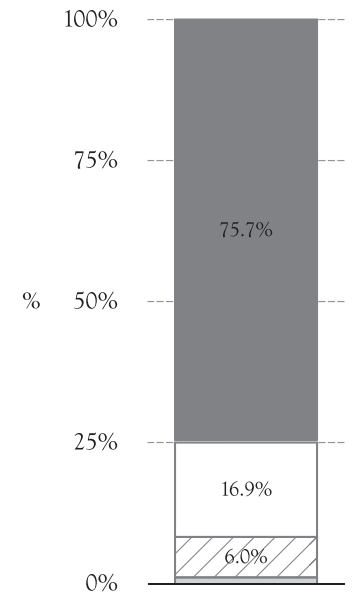

Todos los dias

$\square$ Entre 4 y 5 dias por semana

$\square$ Entre 2 y 3 dias por semana

$\square$ Un dia por semana
Panel b: dias de la semana empleado en mototaxismo

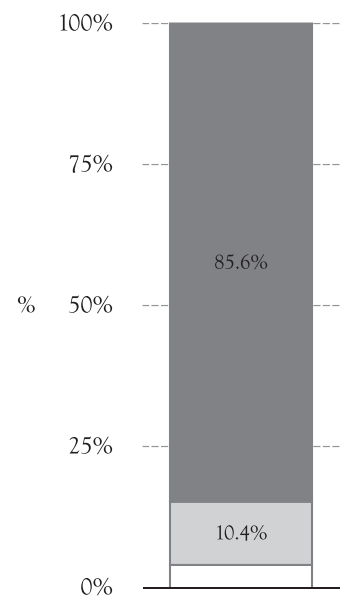

Todos los dias

Fines de semana o festivos

De lunes a viernes

Fuente: Elaboración propia, a partir de cuestionario aplicado a mototaxistas. 


\section{GRÁFICO 5 \\ Exclusividad para ejercer el oficio. Mototaxistas de Cartagena}

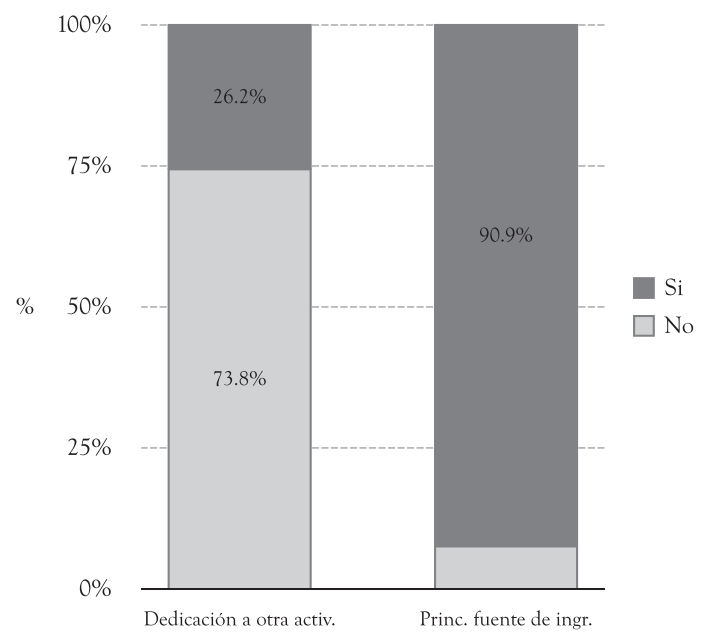

Fuente: Elaboración propia, a partir de cuestionario aplicado a mototaxistas.

En cuanto a las razones por las cuales las personas se dedican al oficio de mototaxistas, el $74,94 \%$ indican que la principal obedece a la dificultad para encontrar un trabajo u oficio alternativo. De hecho, fueron pocos los que respondieron que lo hacían porque fuese bien remunerado $(24,07 \%)$, les generase ingresos adicionales $(10,92 \%)$ o porque les gustase $(9,4 \%)$ (Gráfico 6).

En su actividad, la mayoría de mototaxistas posee moto propia $(67,74 \%)$, aunque un $26,05 \%$ de ellos alquila o renta la moto para el trabajo, y sólo un $6,20 \%$ de ellos usan motos prestadas. Para el $74,25 \%$ de los conductores que alquilan este tipo de vehículo, la tarifa de alquiler oscila entre $\$ 16.000$ y $\$ 20.000$ por día (Cuadro 3).

La tarifa de arrendamiento termina siendo un valor bajo, si se tienen en cuenta las utilidades del oficio, que suelen ser de más del 50\% de los costos de alquiler. De hecho, la utilidad para el 23,88\% de los mototaxistas -después de descontar todos los costos asociados a la operación del vehículo- oscila entre los $\$ 55.001$ y $\$ 65.000$ diarios (Gráfico 7), cifra superior al salario mínimo diario legal vigente en Colombia para 2018, que se situó en $\$ 26,041.40$ aproximadamente (Banco de La República, 2018). Vale la pena indicar que no existe una asociación significativa entre la utilidad y la propiedad de la motocicleta. ${ }^{4}$

\footnotetext{
${ }^{4}$ Prueba $\chi 2$, con un nivel de significancia del 5\% y 18 grados de libertad; P-valor propiedad=0,13.
} 


\section{GRÁFICO 6}

Razones para elegir dedicarse al ejercicio de mototaxista en Cartagena

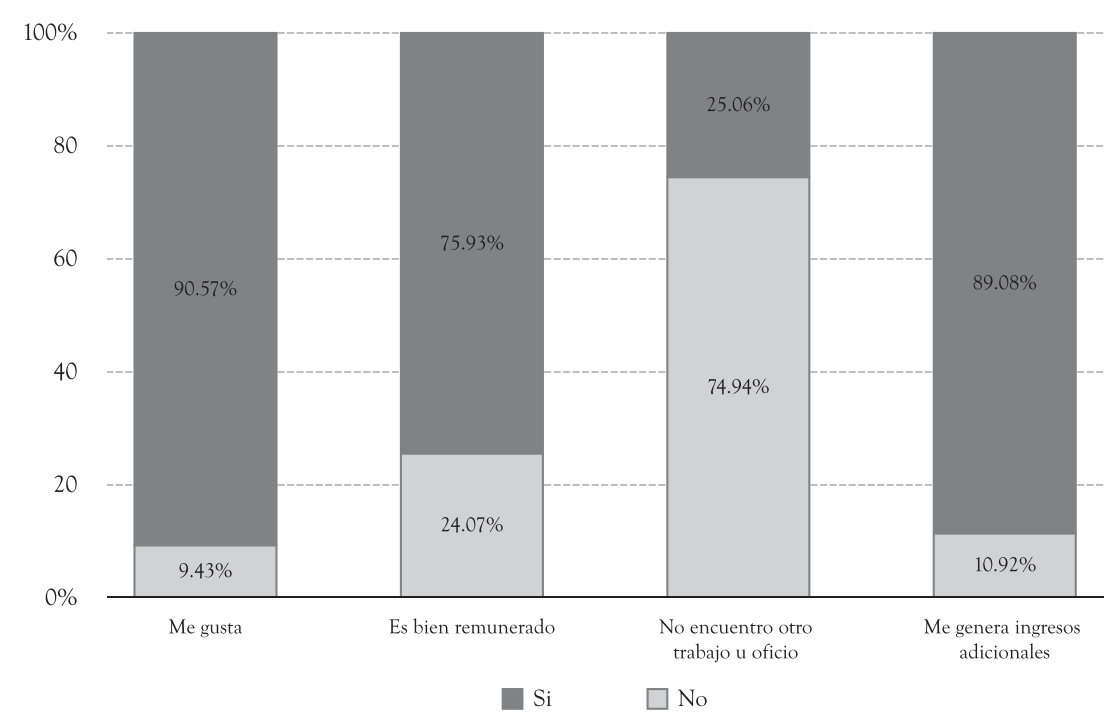

Fuente: Elaboración propia, a partir de cuestionario aplicado a mototaxistas.

\section{CUADRO 3}

Propiedad de la motocicleta y tarifa de alquiler. Mototaxistas de Cartagena

Panel a: propiedad de la motocicleta

\begin{tabular}{lc}
\hline Tipo de propiedad & $\%$ \\
\hline Propia & $67.7 \%$ \\
Alquilada & $26.1 \%$ \\
Prestada & $6.2 \%$ \\
\hline
\end{tabular}

Panel b: tarifa de alquiler

\begin{tabular}{lr}
\hline \multicolumn{1}{c}{ Tarifa } & $\%$ \\
\hline Entre $\$ 10.000$ y $\$ 15.000$ & $19.8 \%$ \\
Entre $\$ 16.000$ y $\$ 20.000$ & $74.3 \%$ \\
Más de $\$ 20$ mill & $6.0 \%$
\end{tabular}

Fuente: Elaboración propia, a partir de cuestionario aplicado a mototaxistas. 


\section{GRÁFICO 7 \\ Utilidades diarias. Mototaxistas de Cartagena}

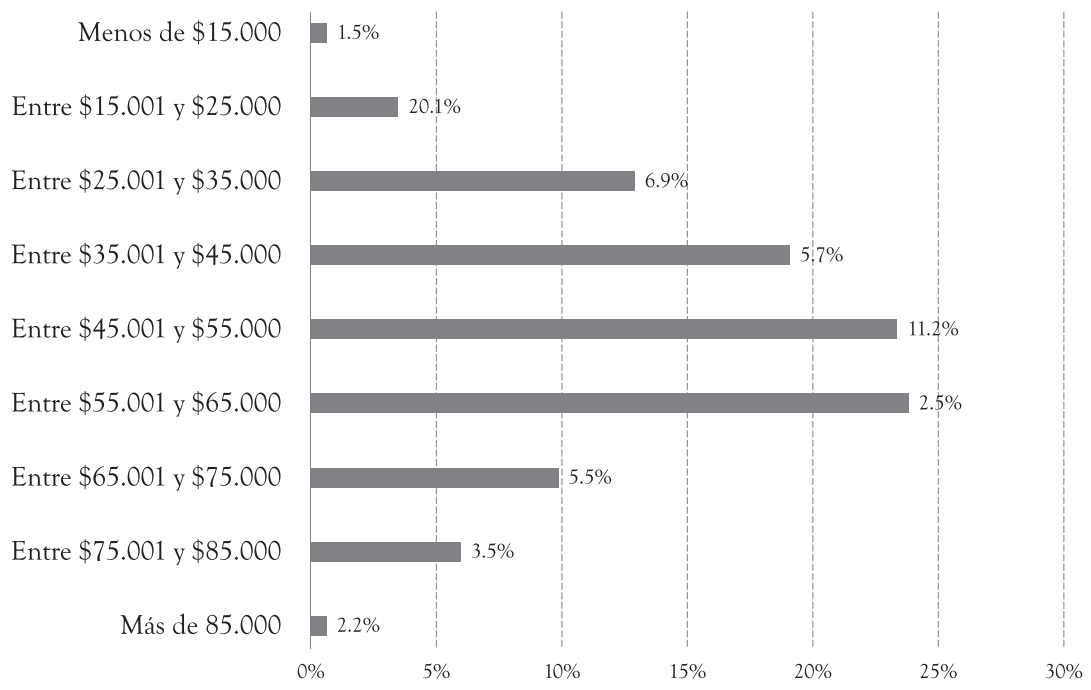

Fuente: Elaboración propia, a partir de cuestionario aplicado a mototaxistas.

Las utilidades obtenidas del oficio de mototaxismo son usadas tanto para pagar el sostenimiento personal del conductor como para cubrir las necesidades de las personas que tienen a su cargo. De hecho, el 74,46\% de quienes ejercen el oficio tienen la responsabilidad de sostener a sus familias, generalmente integradas por entre dos y cuatro miembros. Es de anotar que el parentesco de los miembros bajo su responsabilidad es muy variado: pareja e hijos, padres o suegros, entre otros (Maza et al. 2018) (Gráfico 8).

En general, la mayoría de mototaxistas llevan entre 0 y 4 años dedicándose al oficio; de ellos, un porcentaje considerable lo ejerce todos los días de la semana, porque es su principal actividad generadora de ingresos, lo cual podría decirse que obedece a la falta de oportunidades para encontrar otro empleo. Gran parte de estas personas poseen su propia moto para trabajar, y todas sus decisiones están motivadas por la necesidad que tienen de generar ingresos al ser la cabeza responsable de su hogar. 


\section{GRÁFICO 8}

Responsabilidad como cabeza de familia y personas a cargo.

Mototaxistas de Cartagena

Panel a: responsabilidad como cabeza de familia

\begin{tabular}{cc}
\hline Cabeza de familia & $\%$ \\
\hline Sí & $74.5 \%$ \\
No & $25.5 \%$ \\
\hline
\end{tabular}

Panel b: número de personas a cargo

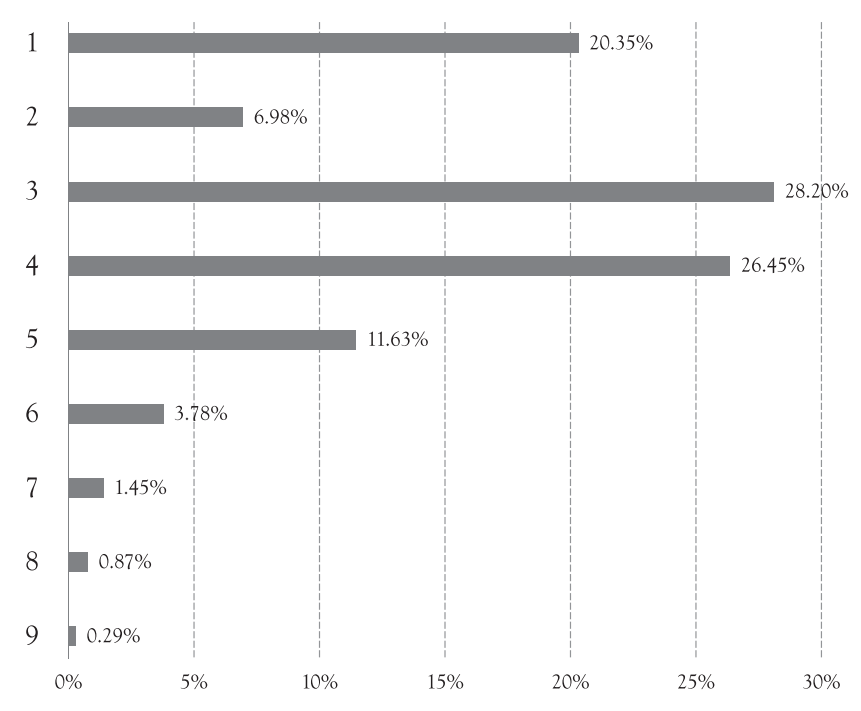

Fuente: Elaboración propia, a partir de cuestionario aplicado a mototaxistas.

\section{B. Factores de riesgo del oficio de mototaxismo en Cartagena}

Antes de explorar los factores de riesgo del oficio de mototaxismo en Cartagena, cabe resaltar que un 55,33\% de ellos dedican jornadas de trabajo mayores a la máxima laboral diaria que es de 10 horas, según lo contempla el artículo 161 del Código Sustantivo del Trabajo, lo que obedece a la necesidad de satisfacer la gran demanda del servicio, y a su vez, cubrir las necesidades de ingresos a los mismos conductores (Gráfico 9). 


\section{GRÁFICO 9 \\ Horas dedicadas al oficio del mototaxismo}

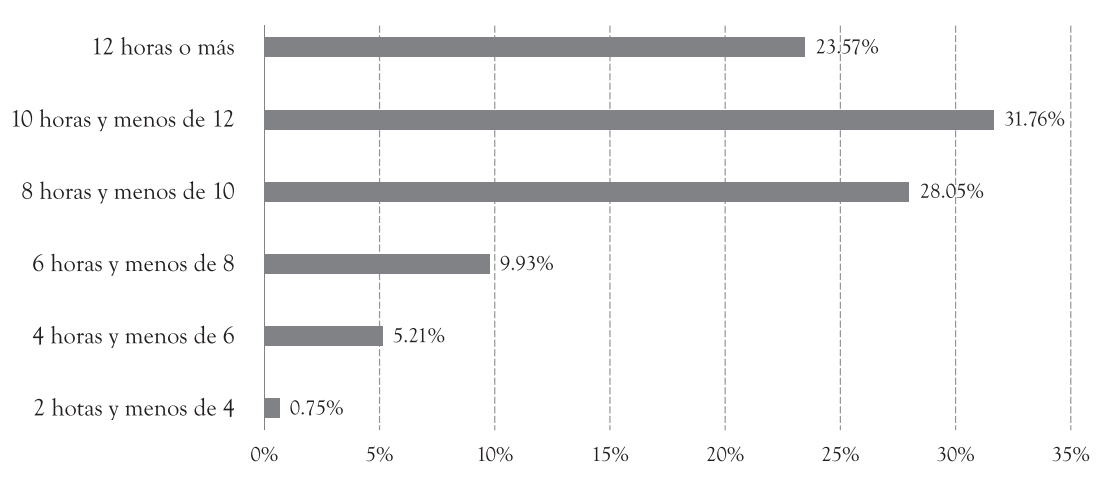

Fuente: Elaboración propia, a partir de cuestionario aplicado a mototaxistas.

Para este caso de estudio, se tomaron factores de riesgo desde los puntos de vista ambientales, físicos y psicológicos. En cuanto a factores de riesgo ambientales, se destacan exposiciones a radiación solar y altas temperaturas. Para ambos factores de riesgo existe una alta valoración que equivale a mucho más de la mitad en cuanto al nivel de exposición, por lo que tanto las radiaciones solares, como las altas temperaturas se convierten en un factor de riesgo muy influyente y considerable en cuanto a la salud de quienes practican el mototaxismo en Cartagena (Gráfico 10).

Los factores de riesgo físicos fueron un elemento influyente, puesto que las posiciones, posturas, y los perjuicios que trae consigo la informalidad del oficio, no se evitan con el uso instrumentos de protección, ni mucho menos con el método de ejercer el oficio. La seriedad de este riesgo se refuerza en la respuesta a la pregunta del cuestionario en la que una proporción alta (72\%) de los mototaxistas afirmaron tener o haber tenido molestias por la posición al conducir.

En cuanto a factores de riesgo psicológicos, se evaluaron el rechazo y percepción de inseguridad (hurto). En el aspecto de seguridad, el factor obtiene un valor cercano al $95 \%$, de los puntajes más altos de la medición, lo que sugiere una percepción de la gravedad de este problema al que están expuestos durante el día a día, y que claramente está fuera de su control. A esto hay que sumarle que el rechazo a la inseguridad principalmente viene de parte conductores de buses/SITM o taxis $(66,67 \%)$, seguido de conductores de vehículos particulares 
y autoridades de tránsito cuyas proporciones son $61,20 \%$ para ambos casos (Cuadro 4).

\section{GRÁFICO 10}

Valoración de exposición a riesgos ambientales

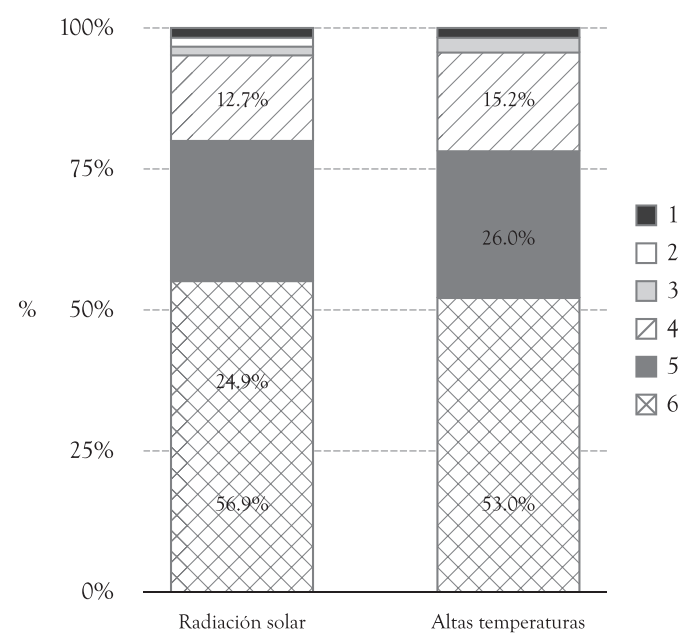

Fuente: Elaboración propia, a partir de cuestionario aplicado a mototaxistas.

\section{CUADRO 4}

Valoración exposición a inseguridad (hurto)

\begin{tabular}{|c|c|}
\hline Escala & $\%$ \\
\hline 0 & $2.23 \%$ \\
\hline 1 & $0.99 \%$ \\
\hline 2 & $1.99 \%$ \\
\hline 3 & $4.96 \%$ \\
\hline 4 & $8.93 \%$ \\
\hline 5 & $80.89 \%$ \\
\hline
\end{tabular}

Fuente: Elaboración propia, a partir de cuestionario aplicado a mototaxistas. 
Dentro de los demás factores de riesgo ambientales, el polvo, el ruido y la contaminación predominan entre los que más se encuentran expuestos al practicar el mototaxismo, y son aspectos ya relacionados con las políticas ambientales de Cartagena. Además, factores de difícil control como la iluminación y el viento tuvieron porcentajes de valoración altos, pero para se pueden mitigar si los usuarios adoptasen medidas de protección (Gráfico 11).

\section{GRÁFICO 11}

Valoración exposición a otros factores ambientales

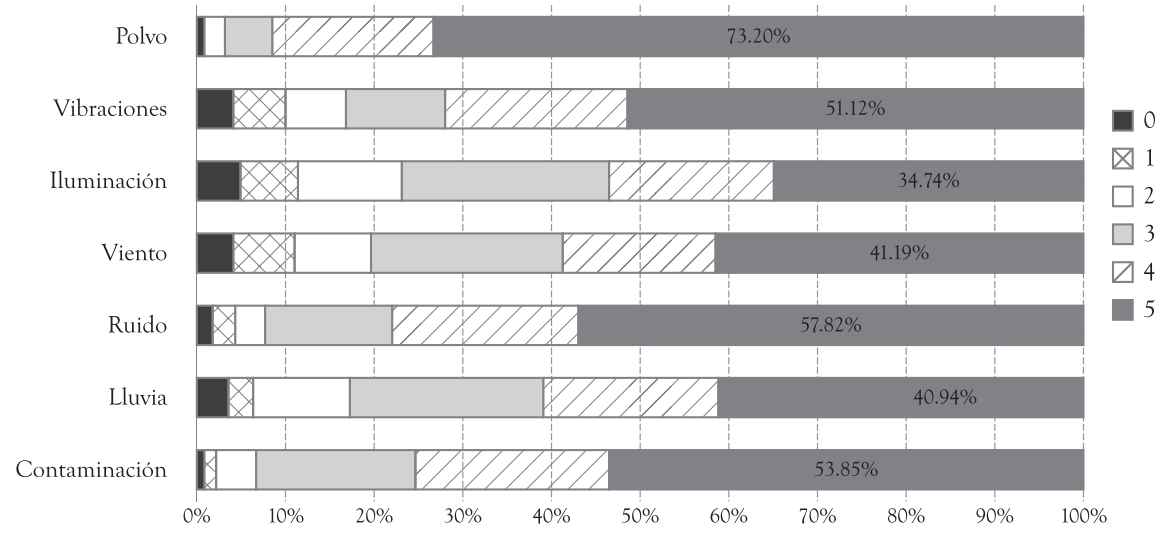

Fuente: Elaboración propia, a partir de cuestionario aplicado a mototaxistas.

\section{Apreciaciones sobre lesiones causadas en el ejercicio del oficio de mototaxismo en Cartagena}

En este apartado se describen las percepciones que tienen las personas que ejercen el oficio de mototaxista en Cartagena sobre las lesiones que puede causar. En términos generales, los mototaxistas son conscientes de los riesgos a los que están expuestos al ejercer el oficio (el 76,62\% lo califican sobre 5, en una escala que va de 0 a 5 , es decir 5 es el riesgo máximo). De igual forma, tienen el conocimiento de que estos accidentes podrían ocasionar lesiones (el 69,98\% lo califican sobre 5), siendo igual de significativas las calificaciones frente a los riesgos que enfrenta esta actividad informal (Gráfico 12). 


\section{GRÁFICO 12}

Percepción sobre la exposición a los accidentes y a lesiones ejerciendo el oficio.

\section{Mototaxistas de Cartagena}

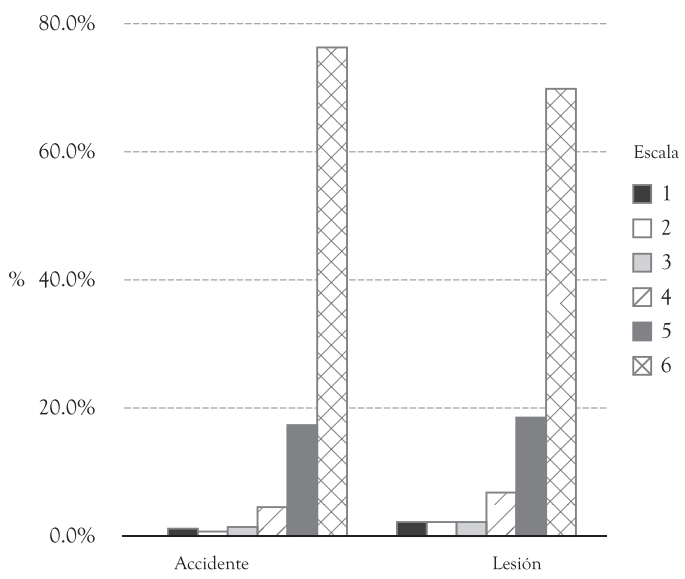

Fuente: Elaboración propia, a partir de cuestionario aplicado a mototaxistas.

\section{GRÁFICO 13}

Percepción de los mototaxistas con respecto a la satisfacción que les genera el oficio. Mototaxistas de Cartagena

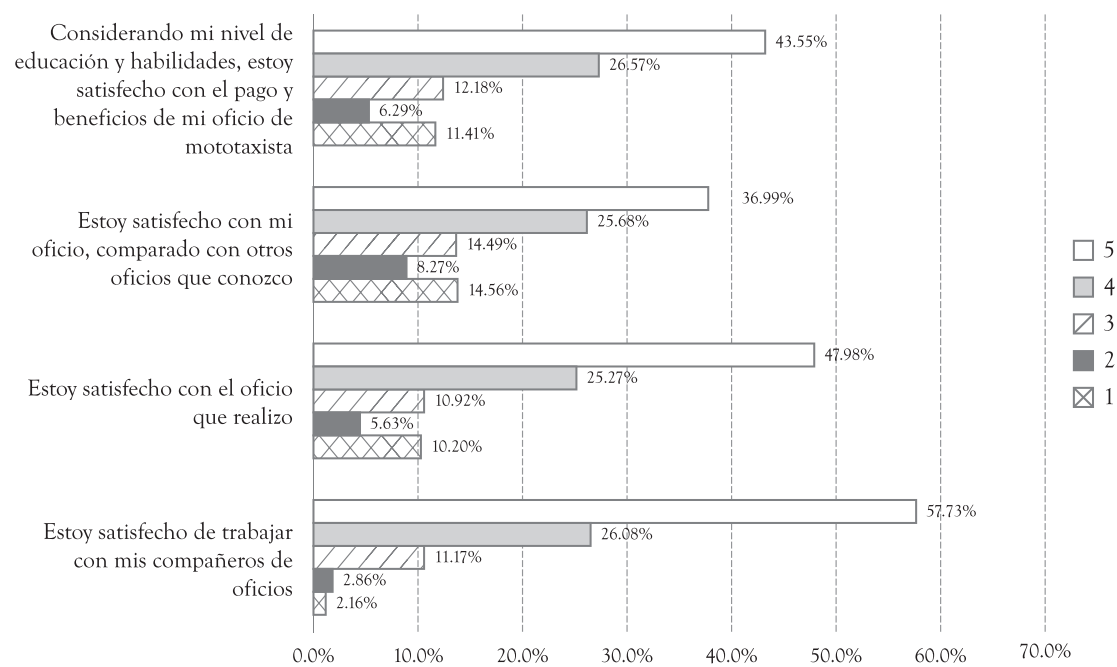

Fuente: Elaboración propia, a partir de cuestionario aplicado a mototaxistas. 
La percepción de satisfacción de los mototaxistas fue alta $(73,25 \%$ de ellos respondió en altos niveles). Al enmarcar la misma pregunta por fuente de satisfacción se obtuvo que este nivel fue de $83,81 \%$, al preguntar por la percepción de trabajar con sus compañeros de oficio, de 62,67\% al compararlo con otras actividades laborales y, por último, de 70,12\% al preguntar por la percepción de si el pago y los beneficios que obtienen del mototaxismo concuerdan con su grado de educación y habilidades (Gráfico 13).

\section{CONCLUSIONES}

Este artículo tuvo como objetivo principal analizar los factores de riesgo asociados al oficio de mototaxistas en Cartagena de Indias, así como la apreciación de lesiones causadas por el tránsito por parte de quienes se dedican a esta actividad. Para alcanzar dicho objetivo, se llevó a cabo un estudio de tipo explicativo de los factores de riego y percepciones sobre lesiones causadas por el tránsito, a una muestra representativa de la población de mototaxistas de Cartagena.

Los resultados muestran que el oficio de mototaxismo se ha constituido tanto en una oportunidad de generación de ingresos para quienes los practican, como en una buena opción de movilidad para los usuarios, en términos de rapidez y eliminación de barreras físicas que limitan a otros medios de transporte. Sin embargo, dicho oficio es considerado un perjuicio, porque pone en riesgo la integridad física y mental tanto de conductores como de pasajeros, además de afectar la movilidad de la ciudad, debido a la informalidad, la poca regulación y las precarias condiciones del tipo de transporte. En general, en este oficio predominan los adultos jóvenes, de edad entre 18 y 34 años, cuyo porcentaje más alto con respecto al estrato socioeconómico es el correspondiente al Estrato 1. En su mayoría llevan pocos años ejerciendo el oficio, se dedican mayormente toda la semana a ejercer su oficio, y asimismo el dedican jornadas de trabajo mayores a la jornada máxima laboral diaria que es de 10 horas. Dentro de las razones expuestas la principal obedecía a la dificultad para encontrar un trabajo u oficio alternativo.

Por otra parte, el mototaxismo una actividad que exige a las personas que lo desempeñan realizar esfuerzos físicos y mentales, por ello estos se encuentran constantemente expuestos a altos niveles de riesgos ambientales (radiación solar, altas temperaturas, polvo, vibraciones, ruido, lluvia, contaminación), físicos (posturas y/o movimientos incomodos) y psicológicos (rechazo e inseguridad): todos estos factores son posibles causas de afectaciones en su salud de esta 
población. Con respecto a la accidentalidad, se percibe un bajo reconocimiento y, con ello la concepción de los daños como "gajes del oficio", aunque algunos de estos pueden ocasionar secuelas permanentes, no determinan el abandono de la labor.

En cuanto a la percepción de las lesiones causadas de la población de mototaxistas, un gran porcentaje de ellos reconocen que este oficio representa un gran riesgo, además de estar expuestos a accidentes de tránsito, también estos podrían causar lesiones; sin embargo, se ven en la obligación de continuar dedicándose a este oficio al no tener otras oportunidades de conseguir empleo, y teniendo la necesidad de generar ingresos para sostener a sus familias. No todos se encuentran felices con desempeñar esta labor, pero resaltan factores no del todo negativos. Por ejemplo, consideran a sus compañeros como parte de su familia y creen que los ingresos que reciben desarrollando esta actividad van de acuerdo con sus estudios y habilidades.

Frente los resultados anteriores, y entendiendo la importancia de las medidas de seguridad vial como garantía de la vida, se sugiere a las autoridades de tránsito y transporte de Cartagena fortalecer los mecanismos de seguimiento y control a la seguridad vial, necesarios para contrarrestar el aumento de la accidentalidad vial, producto de la tendencia de crecimiento acelerado del parque automotor, en especial, el de motocicletas. También se sugiere implementar programas encaminados a fortalecer la educación vial, donde las autoridades de tránsito promuevan espacios de sensibilización para motivar el respeto a las señales de tránsito por parte de todos los actores de la vía, así como reforzar los consejos en materia de seguridad vial. Estos programas también deben incluir la formación de niños y jóvenes desde las escuelas, pues ellos se convertirán en los futuros actores viales. Es fundamental seguir fortaleciendo las campañas de disuasión al uso de la moto como medio de transporte público e insistir en la priorización del Plan Vial de Bolívar, para frenar el incremento de la siniestralidad vial y la mortalidad en esa zona del norte colombiano. Vale señalar, no obstante, que el éxito de las medidas a implementar requerirá de una evaluación de la efectividad de las estrategias que las autoridades han implementado hasta el momento, para así evitar incurrir en errores de planificación.

\section{REFERENCIAS}

Barbosa, Kevan Guilherme., Lucas-Neto, Alfredo., Gama, Bruno., LimaNeto, Jose., Lucas, Rilva Suely y D’Ávila, Sergio (2014), „Injuries and 
absenteeism among motorcycle taxi drivers who are victims of traffic accidents", Journal of Forensic and Legal Medicine, No. 26. https://doi. org/10.1016/j.jflm.2014.03.008

Brieva Paternina, José Luis., Tinoco Cantillo, Ulises Antonio., Restrepo Herrera, José Francisco., y Arango Buelvas, León Julio (2011), El mototaxismo en Sincelejo, un análisis socioeconómico. Sincelejo.

Cartagena Cómo Vamos (2019), Resultados Encuesta de Percepción Ciudadana 2018. Cartagena de Indias: Cartagena Cómo Vamos.

Castillo Ávila, Irma Yolanda., Galarza Herrera, Betty y Palomino Gómez, Hibeth (2013), "Condiciones de trabajo y salud de mototaxistas Cartagena», Salud Uninorte, Vol. 29, No. 3.

Espinosa, Catalina., Rodríguez, Claudia., y Uribe, María Carolina (2008), El impacto socioeconómico del fenómeno del mototaxismo en Montería, Trabajo de grado, Especialización en Gerencia Estratégica, Universidad de la Sabana.

Herazo, Yaneth., Domínguez, Regina., Olarte, Sandra., y Quitian, Liliseth (2011), "Discapacidad y mototaxismo en Cartagena , Colombia», Revista Facultad Nacional de Salud Pública, Vol. 29, No. 4.

Hothem, Zachary., Simon, Robert., Barnes, Wesley., Mohammad, Azmath., Sevak, Shruti., Ziegler, Kahtryn., Iacco, Anthony., y Janczyk, Randy (2017), "Effects of repealing the motorcycle helmet law in Michigan", American Journal of Surgery, Vol. 214, No. 3. https://doi.org/10.1016/j.amjsurg.2016.09.052

Jiménez, Camila., Gavalo, Kiu-Tiki., Tejada, María., Rendón, Sandra., y Pautt, Daniela (2013). Percepción de los estudiantes de la Universidad de Cartagena sobre el mototaxismo. Universidad de Cartagena, Cartagena de Indias.

Madougou, Soumaïla., Chigblo, Pascal., Tchomtchoua, Ariel., Lawson, Eric., Yetognon, L., y Hans-Moevi Akue, Aristote (2016), »Incidence et impacts des accidents de la voie publique chez les conducteurs de taxi-moto en milieu tropical«, Revue de Chirurgie Orthopedique et Traumatologique, Vol. 102, No. 2. https://doi.org/10.1016/i.rcot.2016.01.005

Maza Ávila, Francisco Javier., Fals Galezo, María Paula., Licona Dáger, Daniela., Espinoza Flórez, Laura Cristina., y Safar Cano, Camila (2019), Factores de riesgo asociados a la accidentalidad entre mototaxistas de Cartagena de Indias. Cartagena de Indias: Universidad de Cartagena. Mimeo.

Maza Ávila, Francisco Javier., Daniels Puello, Amaranto de Jesús., Blanco Bello, Rosario., y Vergara Schmalbach, Juan Carlos (2017), El transporte informal en los municipios de Arjona, Turbaco, El Carmen de Bolívar y 
Magangué, departamento de Bolívar. Cartagena de Indias. Mimeo.

Quiñones-Bolaños, Edgar Eduardo., Bustillo-Lecompte, Ciro Fernando., y Mehrvar, Mehrab (2016), "A traffic noise model for road intersections in the city of Cartagena de Indias, Colombia«, Transportation Research Part D: Transport and Environment, Vol. 47.https://doi.org/10.1016/j.trd.2016.05.007 Quistberg, Alex., Koepsell, Thomas., Boyle, Linda., Miranda, Jaime., Johnston, Brian., y Ebel, Beth (2014), »Pedestrian signalization and the risk of pedestrian-motor vehicle collisions in Lima, Peru«, Accident Analysis and Prevention, No. 70. https://doi.org/10.1016/j.aap.2014.04.012

Parra Arias, Zunilda., y Barrientos, E. Guilarte (2006), „El transporte informal en ciudades de mediano porte: La motocicleta en Santiago de Cuba«, Transporte Desarrollo y Medio Ambiente, Vol. 26, No. 1.

Sánchez Jabba, Andrés (2011), La economía del mototaxismo: el caso de Sincelejo. (Documento de Trabajo sobre Economía Regional No. 140). Cartagena: Centro de Estudios Económicos Regionales del Banco de la República.

Yánez Contreras, Martha y Acevedo González, Karina (2014), „Determinantes de la decisión de los mototaxistas en Cartagena (Colombia) de infringir la medida de pico y placa«, Revista Facultad de Ciencias Económicas, Vol. 22, No. 1. https://doi.org/10.18359/rfce.638 